\title{
A study on impact of medicinal plants Polyalthia longifolia and Bacopa monnieri with reference to acne treatment
}

\author{
Esther Shoba R [1], Allwin Michael [2], Samuel Reinhard T [3] \\ Department of Life Sciences, Kristu Jayanti College (Autonomous), K. Narayanapura, Kothanur (PO), Bengaluru - 560077 \\ email: esther@kristujayanti.com
}

\begin{abstract}
One of the prerequisites for the success of primary health care is the availability and use of suitable drugs. Plants have always been a common source of medicaments, either in the form of traditional preparations or as pure active principles. Awareness of medicinal plants usage is a result of the many years of struggles against illnesses due to which man learned to pursue drugs in barks, seeds, fruit bodies, and other parts of the plants. Nowadays, researchers more than before are dependent on medicinal plants for discovery of new drugs with fewer side effects. Hence, it is need of the hour to screen for such novel plant sources and bring out its unexplored medical applications to cure and prevent lifestyle diseases like Acne. In this study, plant components were isolated and were studied against propionic bacteria, which cause acne in humans.
\end{abstract}

Keywords Acne, Propionibacterium, medicinal plants

\section{Introduction}

Acne vulgaris is a chronic inflammatory disorder of the sebaceous follicles. Propionibacterium acnes plays a critical role in the development of these inflammatory lesions. Normal skin commensals including Propionibacterium acnes, Staphylococcus epidermidis, Streptococcus pyrogenes and Staphylococcus aureus, proliferate rapidly during puberty and are often involved in the development of acne $(1,2)$. P. acnes is a Grampositive anaerobic bacterium that mostly resides in the pilosebaceous follicles of the skin. Acne is a chronic, inflammatory disease of the pilosebaceous unit estimated to affect $9.4 \%$ of the global population. A small study of adults with acne found clinically significant anxiety and depression in $44 \%$ and $18 \%$ of the sample, respectively (3) and a large survey of 18 -year-olds found that participants with acne had significantly more depressive symptoms, lower self-attitude and selfworth, more feelings of uselessness, and lower body satisfaction than those without acne (4). It has also been widely accepted that inflammatory acne induced by host immune reactions to acnes releases chemo active factors that attract immune system cells such as neutrophils, monocytes, and lymphocytes (4). Studies have found that $P$. acnes stimulate the production of proinflammatory cytokines such as interleukins 1,8,12 and tumor necrosis factor- $\alpha$ (TNF- $\alpha$ ) (5). Sebaceous follicles provide an ideal anaerobic lipid-rich environment for $P$. acnes. It has been proposed that $P$. acnes may exert an effect on naive CD4 cells, initiating their transformation into $T$ helper (TH) 17 cells; this result in the production of IL-17, which is expressed in acne lesions $(6,7)$. Both vitamins $A$ and D could be effective tools in modulating TH 17-mediated diseases such as acne; however, the relevance of IL- 17 in the pathogenesis of acne requires further elucidation, and its importance both clinically and epidemiologically is not yet known. (8). In inflammatory acne lesions, $P$. acnes phylotype IA has been found to be increased, while phylotypes IB and type II are decreased.

As a family of skin disorders, acne is one of the most prevalent dermatologic diseases in the world. It usually affects almost everybody during the life. The use of natural remedies dates back thousands of years. It is estimated that there are 250,000 to 500,000 species of plants on Earth (9). Tetracycline, erythromycin, roxithromycin, clindamycin, benzoyl peroxide, and azelaic acid are some examples of these antibiotics. However, antibiotic resistance has been increasing in prevalence within the dermatologic setting (10). To overcome the problem of antibiotic resistance, natural products have been extensively studied as alternative treatments for diseases. The development of antibiotic resistance is multifactorial, involving the specific nature of the relationship of bacteria to antibiotics. Therefore, there are sufficient purposes for searching alternative remedies that work out and resolve these problems. The medicinal properties of several herbal plant species have been documented in ancient Indian literature and the formulations had been found to be effective in treating various diseases. Therefore to meet the increasing demand for manufacturing modern medicines and their export, the need for medicinal plants have enormously increased. In addition to it because of the high treatment cost, medicinal plants have been studied as alternative treatments for diseases. As an alternative approach, numerous reports have indicated the possibility of using medicinally potent plant actives to counter the growth of the bacteria and its inflammatory response. The present study is an attempt to investigate the medicinal property of plants Polyalthia longifolia and Bacopa monnieri with reference to acne treatment.

\section{Materials and Methods}




\section{Collection of plant sample}

Bacopa monnieri and Polyalthia longfolia plant samples were collected from the medicinal garden of Kristu Jayanti College (Autonomous). Aerial parts of both the plants were selected for the study. These plant samples were thoroughly washed and then kept for shade drying for almost a week and preceded for extraction The samples were extracted using both warm and cold extraction methods.

\section{Phytochemical Analysis}

Preliminary screening of phytochemicals was carried out in all the extracts and chief phytoconstituents of the selected medicinal plants were identified in order to relate their presence with bioactivities of the plants. Tests for tannins, flavonoids, terpenoids, saponins, steroids, phlobatannins, carbohydrates, glycosides, alkaloids, proteins, anthroquinonens using standard methods.

\section{Antimicrobial susceptibility testing}

\section{Culturing of Propionibacterium acnes}

Authenticated culture of Propionibacterium acnes was procured from St. Johns National Academy of Health Sciences, Bengaluru and sub culturing was done on blood agar medium by incubating at $37^{\circ} \mathrm{C}$ in anaerobic conditions for next 48 hours.

\section{Well diffusion method}

This experiment was carried out by the method of Hayes and Markovic (2002) with some modifications. P. acnes was incubated in liquid Reinforced Clostridium Medium (RCM) for $48 \mathrm{~h}$ under anaerobic conditions and adjusted to yield approximately $1.0 \times 10^{8} \mathrm{CFU} / \mathrm{ml}$. The inoculum was spread on blood agar medium plates. The plates were well punctured with $6 \mathrm{~mm}$ diameter. Plant extract of concentrations ranging from 50 to 200 $\mathrm{mg} / 50 \mu \mathrm{L}$ was added to the wells and the plates were then incubated at $37^{\circ} \mathrm{C}$ for $48 \mathrm{~h}$ under anaerobic conditions (in Gas Pak Jars). Solvent used for plant extraction was used as control.

\section{Determination of minimum inhibitory}

The minimal inhibitory concentration (MIC) values were determined by broth dilution assay (Murray et al., 1995). The cultures used were $48 \mathrm{~h}$ broth culture of $P$. acnes. The MIC was defined as the lowest concentration of the compound to inhibit the growth of microorganisms. A stock solution $(500 \mathrm{mg} / \mathrm{ml})$ of plant extract was prepared in $10 \%$ DMSO. The test tubes were filled with $9.7 \mathrm{ml}$ of media and $0.2 \mathrm{ml}$ of different concentration of extract (50mg, $100 \mathrm{mg}, 150 \mathrm{mg}, 200 \mathrm{mg}, 250 \mathrm{mg}$ and $300 \mathrm{mg}$ ) was added to the 7 test tubes of 10 . One of the remaining three tubes was used as a positive control by adding 0.2 $\mathrm{ml}$ of reference antibiotic solution $(100 \mathrm{mg} / \mathrm{ml})$, whereas the other two tubes were used as negative control by adding $0.2 \mathrm{ml}$ of DMSO to one tube and $0.2 \mathrm{ml}$ sterile water to the other. All tubes were inoculated with $0.1 \mathrm{ml}$ of the test suspension. The tubes were then incubated for $48 \mathrm{~h}$ at $37^{\circ} \mathrm{C}$ in Gas Pak Jars for the anaerobic bacteria (Murray et al., 1995). After incubation, the MIC of each ingredient was determined by visual inspection of the tubes. The lowest concentration of the active ingredient that inhibited growth of the organism, as detected by lack of visual turbidity (matching the negative growth control) was designated the MIC (Baron et al., 1994).

\section{Spectroscopic analysis}

The FTIR analysis was carried out for the extracts to identify the compounds and the functional group. The spectroscopic analysis was carried out at M/s. Leads Clinical Research \& Bioservices Private Limited.

\section{Chromatographic techniques}

Thin layer chromatography was carried out in readymade plates (Merk). For Polyalthia longifolia the mobile phase used was Methanol: Glacial acetic acid: Formic acid: Water in 3:0.9:0.9:0.5 (Gaurav et al, 2014) and Toluene: Ethyl acetate: Formic acid in ratio 5:4:0.2 (Gaurav et al, 2014). For Bacopa monnieri solvents used were: Butanol: Acetic acid: Water in ratio 3.6:6:8 (Gaurav, 2015) Toluene:Ethyl acetate:Methanol:Glacial acetic acid in ratio 3:3.5:2.5:1 (Shahare,2010).

The sheets were then dried. A modification was made to check if any part of the TLC sheet gave encouraging antibacterial activity against $P$. acnes. For that $0.6 \mathrm{OD}$ culture was mixed with nutrient agar and spread as a single layer over the TLC sheets. The extracted compounds were run on TLC plates and incubated and studied for the inhibitory growth and RF value of all the extracts was calculated.

\section{Lc-Ms Analysis}

Liquid Chromatography Mass Spectrometry (LC-MS/MS) is an exceedingly sensitive and specific analytical technique that can precisely determine the identities and concentration of compounds within sample. On checking the results of TLC, further characterisation was done using LCMS analysis and the compounds separated was tested for antimicrobial activity by broth antimicrobial assay.

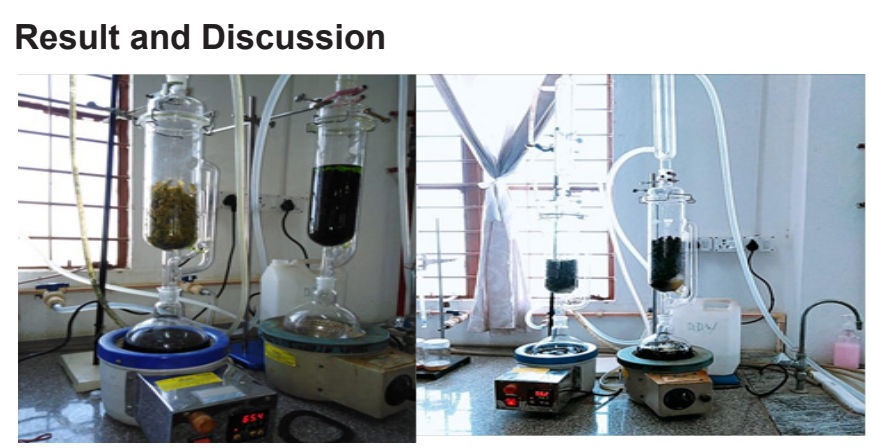

Figure 1: Preparation of plant extracts Polyalthia 
longifolia and Bacopa monnieri is below the sentence Well Diffusion method. Please Take it below the soxhlet apparatus figure.

Among the solvents acetone, diethyether, methanol used for extraction (Figure 1), both the plants Polyalthia longifolia and Bacopa monnieri showed highest phytochemical constituents (Table 1 and 2) in the methanolic extracts as methanol can extract both hydrophilic and lipophilic molecules from plant parts. Well diffusion method

Table 1: Phytochemical analysis of Polyalthia longifolia

\begin{tabular}{|c|c|c|c|c|}
\hline $\begin{array}{c}\text { PHYTO- } \\
\text { CHEMICALS }\end{array}$ & METHANOL & $\begin{array}{c}\text { ETHYLACE- } \\
\text { TATE }\end{array}$ & AQUEOUS & ACETONE \\
\hline Saponins & + & - & + & - \\
\hline Alkaloids & + & - & + & - \\
\hline Tannins & + & - & - & - \\
\hline Glycosides & + & - & - & - \\
\hline Terpenoids & + & - & + & - \\
\hline Flavanoides & + & - & - & - \\
\hline
\end{tabular}

Table 2: Phytochemical analysis of Bacopa monnieri.

\begin{tabular}{|c|c|c|c|c|}
\hline $\begin{array}{c}\text { PHYTO- } \\
\text { CHEMICALS }\end{array}$ & METHANOL & $\begin{array}{c}\text { ETHYL ACE- } \\
\text { TATE }\end{array}$ & AQUEOUS & ACETONE \\
\hline Saponins & + & + & + & + \\
\hline Alkaloids & + & + & + & + \\
\hline Tannins & + & + & + & + \\
\hline Phlobatanins & + & - & + & - \\
\hline Terpenoids & + & + & - & - \\
\hline Flavanoides & + & + & + & + \\
\hline
\end{tabular}

The extracted compounds were tested against $P$. acnes involved in the formation of acne. All the extracts investigated in this study were found to possess marked antibacterial activity against $P$. acnes. Good zone of inhibition was observed for all concentrations of Polyalthia longifolia methanol extract (50 to 200 $\mathrm{mg} / 50 \mu \mathrm{L}$ ) (Figure 2) and minimum for the aqueous extract at a concentration of $50 \mathrm{mg} / 50 \mu \mathrm{L}$ and for Bacopa monnieri highest activity in methanol extract $200 \mathrm{mg} / 50 \mu \mathrm{L}$ and lower with the aqueous extract at a concentration of 50mg/50 $\mu \mathrm{L}$ (Table 3).

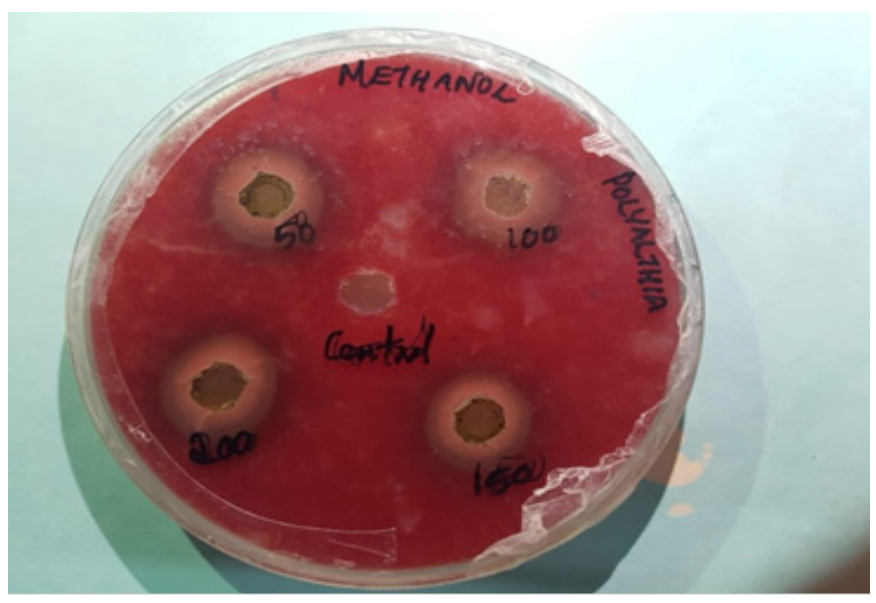

Figure 2: Methanol Extract of Polyalthia longifolia on P.acne

\section{Determination of minimum inhibitory}

Minimum inhibitory concentration was determined to check which plant extract had a stronger antibacterial activity at lower concentrations. In case of Polyalthia longifolia, methanol extract MIC was observed at $50 \mathrm{mg} / 0.2 \mathrm{ml}$, while acetone showed its minimum inhibitory concentration at $150 \mathrm{mg} / 0.2 \mathrm{ml}$ and ethyl acetate at $100 \mathrm{mg} / 0.2 \mathrm{ml}$ respectively. In Bacopa monneri MIC was observed at a concentration of $200 \mathrm{mg} / 0.2 \mathrm{ml}$ for both methanol and acetone extract, whereas for methyl acetate it was observed at $150 \mathrm{mg} / 0.2 \mathrm{ml}$.

\section{Spectroscopic analysis and Chromatographic techniques}

The FTIR analysis was carried out and found out

Table 3: Minimum Inhibitory Concentration of plants extracts against P. acne

\begin{tabular}{|c|c|c|c|c|c|c|c|c|c|c|c|c|c|c|c|}
\hline \multicolumn{10}{|c|}{ Polyalthia longifolia } \\
\hline \multicolumn{10}{|c|}{ METHANOL } & \multicolumn{1}{c|}{ ACETONE } & \multicolumn{3}{c|}{ ETHYL ACETATE } & \multicolumn{3}{c|}{ AQUEOUS } \\
\hline 50 & 100 & 150 & 200 & 50 & 100 & 150 & 200 & 50 & 100 & 150 & 200 & 50 & 100 & 150 & 200 \\
\hline 23 & 28 & 27 & 27 & 19 & 20 & 21 & 22 & 22 & 22 & 24 & 20 & 13 & 15 & 16 & 19 \\
\hline \multicolumn{10}{|c|}{ Bacopa monnieri } \\
\hline 50 & 100 & 150 & 200 & 50 & 100 & 150 & 200 & 50 & 100 & 150 & 200 & 50 & 100 & 150 & 200 \\
\hline 21 & 22 & 22 & 23 & 21 & 22 & 21 & 23 & 23 & 23 & 22 & 22 & 12 & 15 & 16 & 17 \\
\hline
\end{tabular}


that the analysis proved the presence of phytochemicals present in the

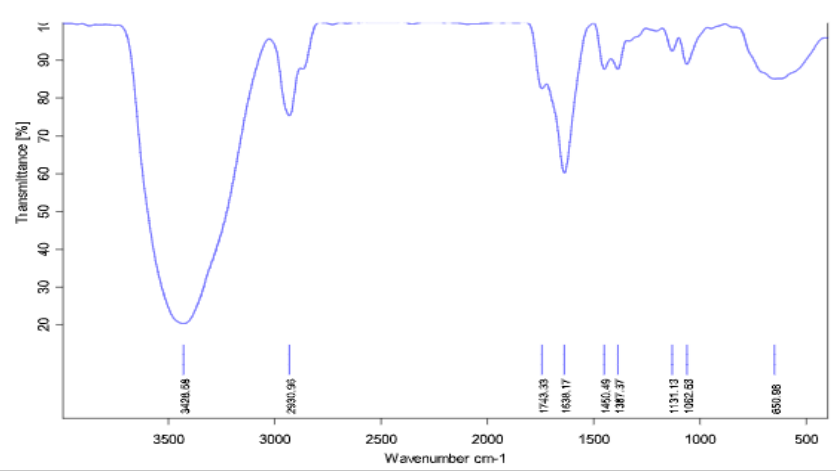

Figure 4: FTIR Analysis of Methanol extract of Polyalthia longifolia

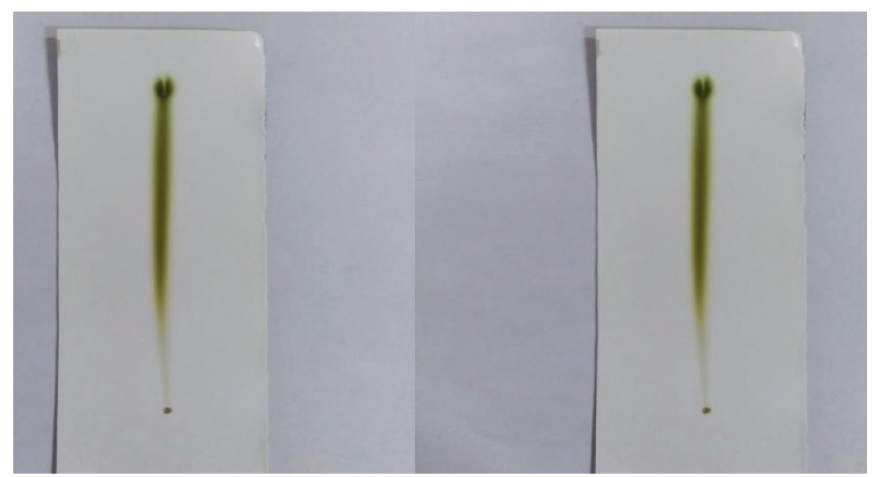

Figure 5: TLC of methanol extract of Polyalthia longifolia against P.acnes

extracts. The methanol extract had highest compounds and ethyl acetate had least compounds in the both extraction. In the methanol extract wave length/ peak
$3428.58 \mathrm{~cm}^{-1}$ and $1638.17 \mathrm{~cm}^{-1}$ in comparison with the standard chart was found to have $\mathrm{O}-\mathrm{H}$ (Alcoholic group) bond and $\mathrm{C}-\mathrm{H}$ Bond (Aromatic compound) (Figure 4). Other extracts showed the presence of Amines and Alkane compounds.

The methanolic extract of Polyalthia longifolia did not support the growth of the bacteria and the RF value of methanol extract of Polyalthia longifolia was found to be $6.3 / 9=0.7$ which is equal to alkaloid compound when comparing to the standard (Figure 5).

\section{LC-MS}

From the LC-MS analysis the potential compounds were separated and found to be Pendulamine, Polylongine and Isooncodine all the three compounds were from alkaloid compounds and each of them was confirmed by Mayer's test (Figure 6, Table 4). These compounds were studied for inhibition of $P$. acne in broth medium. All the potential compounds showed inhibition of $P$.acne where the inhibition was found to be $0.42,0.54$ and 0.58 for Pendulamine, Polylongine and Isooncodine respectively as compared to the control.

\section{Conclusion}

In today's scenario, currently much of the phytochemical research is propagated in higher plants and shrubs. Crude plant extracts were initially assayed for their particular phytoconstituents as well as active fractions. The targets are thought to have immense potential in health care system Plant extracts has shown the presence of various phytoconstituents identified by chromatographic techniques which are therapeutically and economically important. Chromatography has been

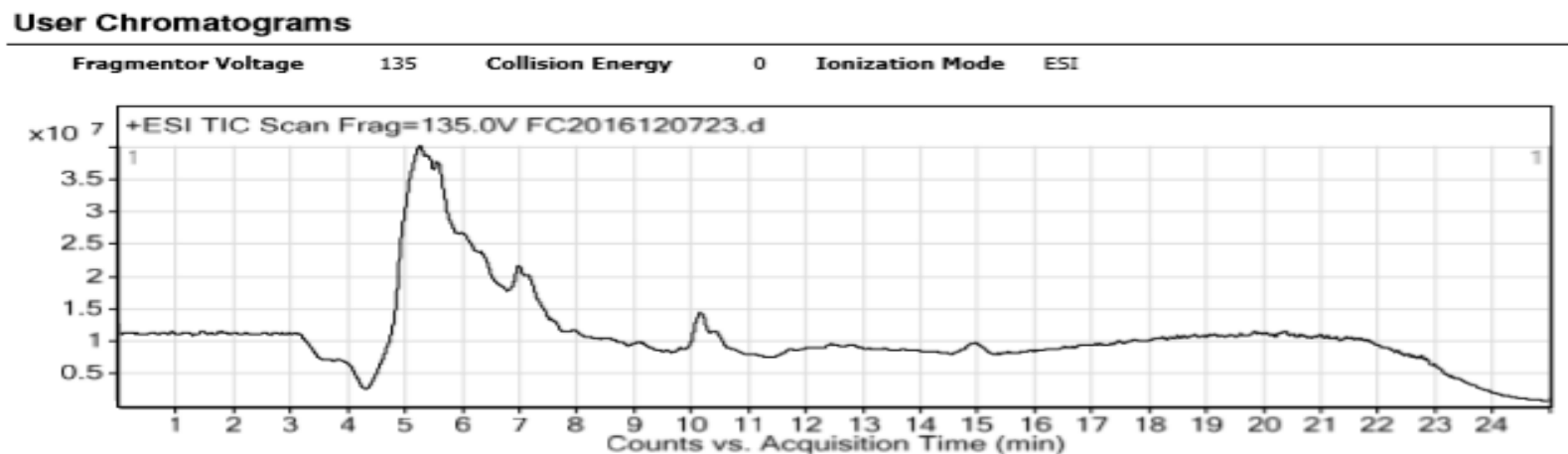

User Spectra

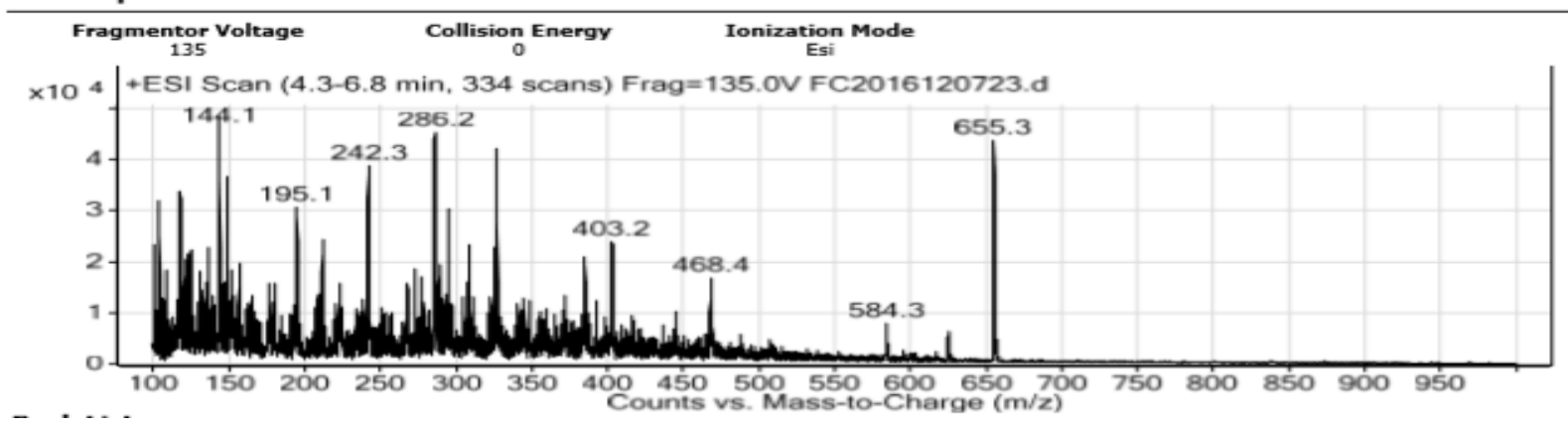

Figure 6: LCMS Chromatogram of methanol extract of Polyalthia longifolia 


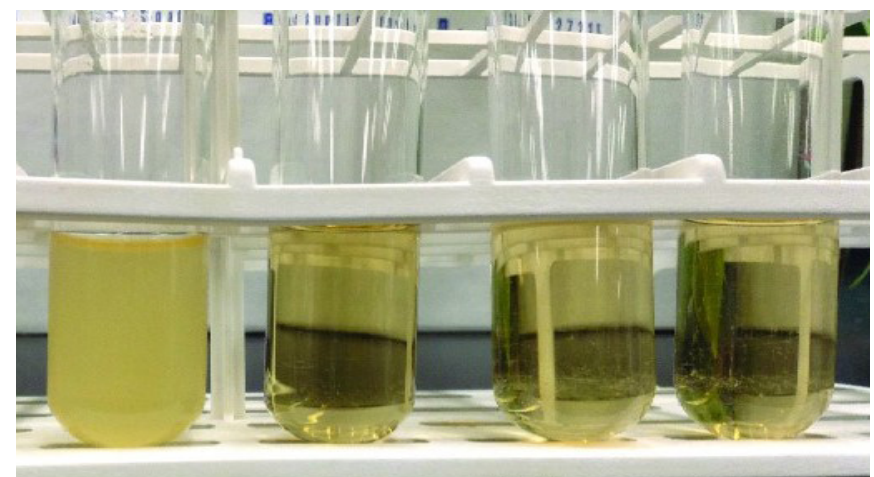

Figure 7: Broth Antimicrobial assay against $P$. acne

regarded as one of the best tool in terms of separation of phytoconstituents by subjecting the extracts to analytical method development. From this study we can conclude that these extract Pendulamine obtained from the methnol extract of Polyalthia longifolia can be used to treat acne individually or in combination.

\section{References}

1. Chomnawang, M.T, Surassmo, S, Nukoolkarn, V.S, Gritsanapan, W. (2005). Antimicrobial effects of Thai medicinal plants against acne-inducing bacteria. J. Ethnopharmacol. 101: 330-303.

2. Nakatuji, T, Kao, M.C, Fang, J.Y, Zouboulis, C.C, Zhang, L, Gallo, R.L, Huang, C.M (2009). Antimicrobial Property of Lauric Acid against Propionibacterium acnes: Its Therapeutic Potential for Inflammatory Acne Vulgaris. J. Invest. Dermatol. 129(10):2480-8.

3. Kellett, S.C, Gawkrodger, D.J. (1999). The psychological and emotional impact of acne and the effect of treatment with isotretinoin. $\mathrm{Br} \mathrm{J}$ Dermatol. 140(2):273-82.

4. Dalgard, F, Gieler, U, Holm, J.O, Bjertness, E, Hauser, S. (2008). Self-esteem and body satisfaction among late adolescents with acne: results from a population survey. J Am Acad Dermatol. 59(5):746-51.

5. Nagy, I, Pivarcsi, A, Kis, K, Koreck, A, Bodai, L, McDowell, A, Seltmann, H, Patrick, S, Zouboulis, C.C, Kemény, L. (2006). Propionibacterium acnes and lipopolysaccharide induce the expression of antimicrobial peptides and proinflammatory cytokines/chemokines in human sebocytes. Microbes Infect. 8: 2195-2205.

6. Agak, G.W, Qin, M, Nobe, J. et al. (2014). Propionibacterium acnes induce an IL-17 response in acne vulgaris that is regulated by vitamin $A$ and vitamin D. J Invest Dermatol. 134:366-73.
IL-17: a key player in the $P$. acnes inflammatory cascade? J Invest Dermatol. 134:307-10.

8. Kwon, H.H, Yoon, J.Y, Park, S.Y, Suh, D.H. Analysis of distribution patterns of Propionibacterium acnes phylotypes and Peptostreptococcus species from acne lesions. Br J Dermatol 2013; 169:1152-5.

9. R P Borris (1996). Natural products research: perspectives from a major pharmaceutical company. J Ethnopharmacol. 51(1-3):29-38.

10. Swanson, J.K. (2003). Antibiotic resistance of Propionibacterium acnes in acne vulgaris. Dermatol. Nurs. 15: 359-362

11. A J Hayes, B Markovic (2002). Toxicity of Australian essential oil Backhousia citriodora (Lemon myrtle). Part 1. Antimicrobial activity and in vitro cytotoxicity. Food Chem Toxicol.40(4):535-43

12. Murray, P.R, Baron, E.J, Pfaller, M.A, Tenover, F.C, Yolke, R.H. (1995). Manual of CInical Microbiology. (6th ed). USA: ASM Press. pp. 1526-1543.

13. Gaurav Mahesh Doshi, Sandeep Prabhakar Zine, Pratip Kashinath Chaskar, and Hemant Devidas Une. (2014). Solicitation of HPLC and HPTLC Techniques for Determination of Rutin from Polyalthia longifolia Thwaites Pharmacognosy Res. 6(3): 234-239.

14. Gaurav Doshi. (2015). High Performance Thin Layer Chromatography and High Performance Liquid Chromatography determination of Quercetin from Polyalthia longifolia leaves. Free Radicals and Antioxidants: 5(2).

15. Shahare M. D., D' Mello P. M, Standardization of Bacopa monnieri and its Formulations with reference to Bacoside $A$, by High Performance Thin Layer Chromatography, International Journal of Pharmacognosy and Phytochemical Research 2010; 2(4):8-12.

7. Thiboutot, D. M, Layton, A.M, Eady, E. A. (2014). 